$\Rightarrow$ COLLOIDS

\title{
Personal space matters
}

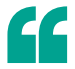

As with systems, the local concentration and structure could be dynamically tunable by temperature, $\mathrm{pH}$ and/or salt concentration biological modifying the

Colloids are useful models for understanding the structure and behaviour of atoms and molecules. Indeed, colloidal phases are governed by many of the same forces that control the interactions between smaller particles. When colloids are confined within a space, the interactions at play are often not rationalizable simply in terms of free-energy minimization. In cylindrical spaces, colloids form particularly interesting assemblies, including helical motifs that mimic spiral patterns in natural systems. Preparing such structures - found in, for example, plant leaves, pine cones, DNA and collagen fibrils - may be key to understanding molecular-scale organization in nature.

Cylindrically confined colloids are typically modelled as non-deformable spheres, the close packing of which is controlled by short-range interactions. However, such hard-sphere

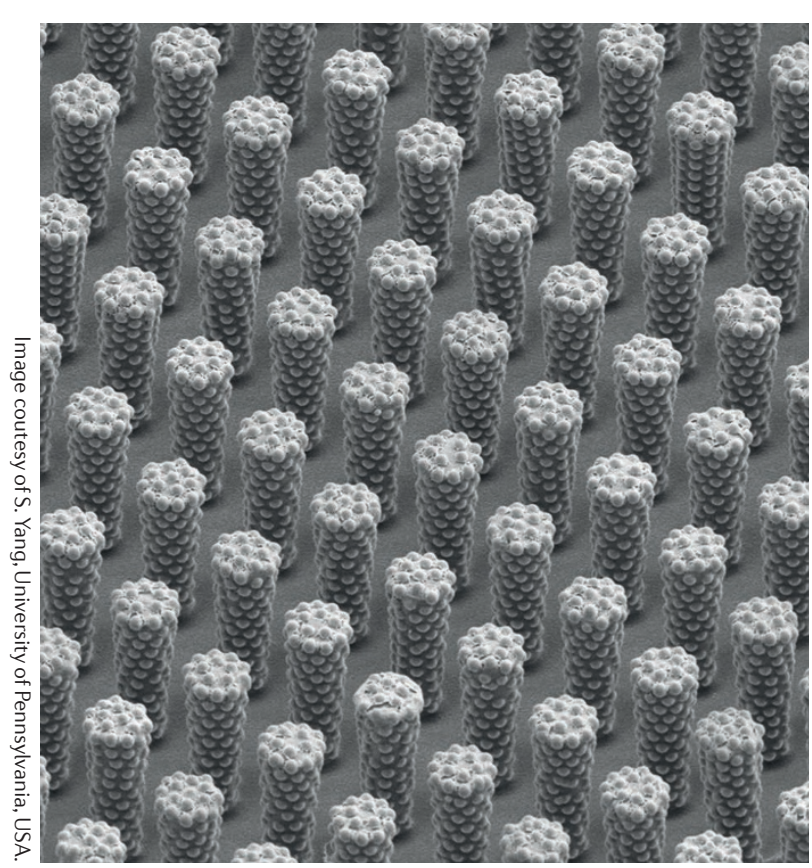

models can be simplistic in that they fail to account for the long-range interactions that commonly dominate biological and macromolecular systems. This deficiency motivated a team led by Shu Yang and Anthony Dinsmore to consider the behaviour of confined 'soft colloids' with long-range repulsions, a study now reported in the Journal of the American Chemical Society.

One problem inherent to the study of soft phases is that it is difficult to image assemblies in situ without disturbing them. The team devised a clever idea: by suspending particles in a photocrosslinkable matrix, they managed to 'freeze' the phases upon confinement. Anionic silica, which bears weakly acidic silanol groups, was selected as the soft-repulsive material. The silica particles were dispersed in trimethylolpropane ethoxylate triacrylate (ETPTA), which has a similar refractive index, resulting in minimization of the van der Waals attractions. Although the diameters of the silica particles are large, so are the length scales of the electrostatic interactions. Notably, the ratio of these values is comparable to that for interactions between molecules.

The suspension was drawn into the cylindrical microchannels of a silicone mold, with rapid curing locking the colloids in place. Extruding the pillars and etching the outer polymer layer to expose the particles yielded an array of free-standing assemblies; the particles within these systems are 'frozen in time' and can be imaged by scanning electron microscopy and focused ion beam.

When hard spheres are confined in a container, they are packed so tightly that their motion is arrested.
There is no space for other particles to squeeze in, and the only way to pack in more particles is to increase the size of the container. The packing landscape for soft colloids is completely different, owing to the overwhelming effects of long-range forces. "In our system, we observe a richer variety of assemblies," says Yang. "The particles repel each other."

The effects of interparticle repulsion were isolated by keeping the channel dimensions constant and increasing the volume fraction of silica particles in ETPTA. As more particles were crammed into the channel, the particles squeezed closer together, and the symmetry of the phase changed as it evolved to accommodate more layers or more particles per layer. The team observed a striking assortment of packing motifs that included achiral phases and chiral helices with three, four or five strands. Confinement is necessary for diversity - when the particles are not confined in microchannels, they adopt a random hexagonal close-packed structure at all concentrations studied.

The team has already begun to add other charged species or responsive molecules to the suspension to make their colloids more closely resemble biological systems. "Proteins and DNA can bear different charges and feature both hydrophobic and hydrophilic moieties such that they commonly give rise to complex and dynamic morphologies," says Yang. "It will be interesting to see how the particle assemblies will be altered. As with biological systems, the local concentration and structure could be dynamically tunable by modifying the temperature, $\mathrm{pH}$ and/or salt concentration."

Ariane Vartanian, Associate Editor, Nature Communications 